\title{
Fundamentals of motion planning for mitigating motion sickness in automated vehicles
}

\author{
Z. Htike, G. Papaioannou, E. Siampis, E. Velenis and S. Longo
}

\begin{abstract}
This paper investigates the fundamentals of motion planning for minimizing motion sickness in transportation systems of higher automation levels. The optimum velocity profile is sought for a predefined road path from a specific starting point to a final one within specific and given boundaries and constraints in order to minimize the motion sickness and the journey time. An empirical approach based on British standard is used to evaluate motion sickness. The tradeoff between minimizing motion sickness and journey time is investigated through multi-objective optimization by altering the weighting factors. The correlation between sickness and journey time is represented as a Pareto front because of their conflicting relation. The compromise between the two components is quantified along the curve, while the severity of the sickness is determined using frequency analysis. In addition, three case studies are developed to investigate the effect of driving style, vehicle speed, and road width, which can be considered among the main factors affecting motion sickness. According to the results, the driving style has higher impact on both motion sickness and journey time compared to the vehicle speed and the road width. The benefit of higher vehicle speed gives shorter journey time while maintaining relatively lower illness rating compared with lower vehicle speed. The effect of the road width is negligible on both sickness and journey time when travelling on a longer road.The results pave the path for the development of vehicular technologies to implement for real-world driving from the outcomes of this paper.
\end{abstract}

Index Terms-Motion sickness; automated vehicles; optimal control; mutil-objective optimization.

\section{INTRODUCTION}

Automated vehicles (AVs) are expected to push towards the evolution of mobility environment in the near future and will have a significant social, economic and environmental impact on the way we live, commute and work. This is due to its ability to reduce traffic accidents, decrease pollution and emissions, improve parking space, reduce traffic congestion, boost productivity, making transportation more affordable and ensuring equal access [1]. However, motion sickness (MS) may severely jeopardize the successful introduction of fully automated vehicles, as well as their acceptance by the public [2]. In reality, a human driver is thought to drive in a manner that prevents motion sickness occurrence, because the driver, who does not want to get carsick, functions as a type of motion sickness detector or predictor [3]. This depicts the main difference between passengers riding on an automated vehicle and a taxi. With the automated vehicles not having this driver's function, the consideration of the minimisation of motion sickness in their implementation and design frameworks, is crucial in order to fully replace the driver and develop the ability to sense as the occupant's motion detector. It is therefore imperative to consider basic perceptual mechanisms in the design process since automated vehicles cannot simply be thought of as living rooms, offices, or entertainment venues on wheels [4].

Motion sickness is a condition marked by symptoms of bodily warmth, sweating, drowsiness, nausea dizziness, and other physical discomfort. It results from a sensory conflict between inputs from the visual, vestibular and somatosensory systems of the human body [5]. The frequency of oscillation provides human sensory system the ability to resolve the motion of the body because the different senses do not all respond to the imposed acceleration (i.e., they have different frequency response) [6]. The low-frequency translational oscillations are mainly responsible for producing conflict between visual and vestibular perceptions of motion [7]. These are particularly between 0.1 to $0.5 \mathrm{~Hz}$ provoking motion sickness while higher frequencies result in vibration discomfort and injury. It is also worth mentioning that low frequency oscillation found in road vehicles are the longitudinal, lateral and yaw acceleration. On the other hand, the heave, roll and pitch motion are found to be more intense at higher frequencies than $0.5 \mathrm{~Hz}[6]$.

In road vehicles, the root cause that leads to most significant motion sickness is the driving dynamics during turns on curvy road, stop-and-go traffic that can cause repeated low frequency lateral and longitudinal accelerations at higher magnitude. In addition, the driving style (i.e., driver behaviour), vehicle speed, route and road type are all the main factors affecting the driving dynamics. However, the effect of the vehicle type on motion sickness is less significant when driving by same driver in a same manner, as the velocity profile would be very similar in all vehicles [7] and [8]. Other factors also include passenger's onboard visual activities, engaging in non-driving task, that arise from the human factors and ergonomics perspective. Considering these factors, susceptibility of motion sickness is expected to increase significantly in self-driving vehicles [2], since handing over the vehicle controls to automation will free up the occupant's time to engage in non-driving task. In addition, the complete transformation of the entire interior into a more leisurely and economically design 
could also take place [9]. For example, the steering wheel, pedal and gear level may be removed, while seating arrangement will also change by adding a table or a working station. As a result of the above, the passengers will be able to engage more and more in either leisurely or economically productive (non-driving) tasks (i.e., reading, watching movies, working and playing games), which will make them unable to anticipate the direction of travel increasing their susceptibility to motion sickness, its severity and its frequency [10]. This makes motion sickness "the elephant in the room" in high-level AVs [11], as more than 2 in every 9 autonomous vehicle passengers are expected to experience motion sickness based on surveys.

Research into motion sickness on automated vehicles has just started to get attention. On contrary to the past where the cause, function, symptoms, and the estimation of motion sickness were the main issues, nowadays the answer to the question of how to minimize motion sickness has arisen. In particular, recent research have focused on providing solutions to mitigate motion sickness with ideas from the field of human factors and ergonomics, interior design, and automotive engineering. In addition, regarding ergonomics, research has focused into seat design [12] and their arrangement [13], active head-tilting [14], cabin lighting, vection (illusion of self-motion) and passenger biometric data collection [15]. In terms of vehicle dynamics, few researchers have focused on comfort from the view of braking control [16], suspension design [17], and adaptive cruise control [18], but limited work has been done in motion planning for vehicle control in terms of motion sickness minimization.

Motion planning is an important part of the decisionmaking system of an automated vehicle taking place after the route planning [19] and the behavioural decision making [20]. The motion planning system is responsible for computing a reference trajectory from the vehicle's starting position to the final position that is dynamically feasible for the vehicle, comfortable for the passenger, and avoid collisions with obstacles detected by the onboard sensors [21]. Most often, the motion planner also minimizes a given objective function particularly in race car dynamics where achieving minimum lap time is the objective [22]. In addition to journey time, the objective function may penalise hazardous motions or motions that cause passenger discomfort. Thus, motion planning is often solved by formulating it as an optimal control problem (OCP) [23]. The OCP finds the continuous control inputs that, considering constraints on controls and states, force the states of a nonlinear system to minimize an objective function defined over a certain interval and for the final state.

Recent studies have emphasized the smooth driving style (i.e., time derivative of acceleration) has been prioritized as one of the main factors in path planning and motion planning problem toward developing selfdriving vehicles [18] and [24]. However, the motion that causes nausea and other physical discomfort to passengers is relatively ignored. Wada [3] proposed a countermeasure to minimize motion sickness by designing velocity profile of self-driving vehicles on a fixed road with different straight and curve regions. From our knowledge, there are examples of motion planning for tilted train [25] and high-speed rail [26]. There is limited work [27], [28], [29] and [30] done that investigates the fundamentals of motion planning in reducing motion sickness in automated road vehicles. However, these work did not fully address the effect of driving dynamics on motion sickness, and the road path did not provide a good representation of real world driving. In addition, the analysis of the trade-off between motion sickness and journey time required further investigation with the application of multi-objective optimization (MOO) method.

In this work, the attention will be turned on motion planning of autonomous vehicles, in respect to minimising motion sickness and journey time using optimal control approach. More specifically, for a predefined road path from a specific starting point to a final destination, the optimum trajectory is sought within specific constraints and given boundary conditions in order to minimize the motion sickness and the journey time. Arguably, it is likely that for minimum sickness the vehicle would move in a slow manner, at constant velocity or come to a stop. Similarly, for minimum journey time, the vehicle would move very fast or at constant acceleration. Therefore, a trade-off between the two components is investigated through MOO by altering the weighting factors. In addition, three case studies are developed to investigate the effect of driving style, vehicle speed, and road width, which are considered among the main factors affecting the driving dynamics which ultimately leads to motion sickness. The correlation between sickness and journey time is represented as a Pareto front. The severity of the sickness is determined using frequency analysis by comparing the low frequency spectra of the accelerations.

\section{Methods \& MATERIAL}

\section{A. Motion Sickness Evaluation}

The most widely used methods for quantifying motion sickness is the empirical method based on British standards (BS 6841-1987) [31] and International standards (ISO 2631-1) [32]. This method has been validated and verified in many literature when compare with the subjective vertical conflict (SVC) theory [33]. The weakness of the empirical approach is that it does not consider habituation as opposed to SVC. This mean for a longer journey time, motion sickness would continue to increase. Although the subjective vertical conflict model has started to gain attention recent years, the accuracy of this model still required more work for validation. The standards describe a method to evaluate motion sickness using empirical approximations. This method is mainly focused for 
vertical motion at sea studies [34]. The method proposes the estimation of motion sickness dose value, $M S D V$, such that higher values correspond to a greater incidence of motion sickness. The $M S D V$ is given by the root mean square of the z-axis acceleration, after it has been frequency weighted:

$$
M S D V_{z}=\left(\int_{0}^{T}\left(a_{z, w}(t)\right)^{2} d t\right)^{\frac{1}{2}}
$$

where $M S D V_{z}$ is the motion sickness dose value for vertical motion (in $\mathrm{ms}^{-1.5}$ ); $\mathrm{T}$ is the total period (in seconds) during which motion could occur; $a_{z, w}(t)$ is the frequency-weight acceleration in vertical direction and it is obtained by applying the relevant frequency weighting filters directly to the time domain acceleration measurements from the vehicle. In the case of low frequency whole body application, $W_{f}$ is used as the frequency weighting filter. For a better understanding of the filters and their application, the standard ISO 8041 [35] should be consulted.

The suitability of this model to predict motion sickness for road vehicle had been examined by Turner and Griffin [7]. After investigating the relationships between vehicle motion and passenger sickness within all axes, they concluded that the model is also suitable for approximating motion sickness in $\mathrm{x}$-axis and $\mathrm{y}$-axis. Therefore, the total motion sickness dose value resulted from lateral and longitudinal motion could be given by Equation (2).

$M S D V_{x y}=\left(\int_{0}^{T} k_{x}^{2} a_{x, w}^{2}(t) d t\right)^{\frac{1}{2}}+\left(\int_{0}^{T} k_{y}^{2} a_{y, w}^{2}(t) d t\right)^{\frac{1}{2}}$

where $M S D V_{x y}$ is the combined motion sickness dose value from lateral and longitudinal motion; $k_{x}$ and $k_{y}$ are the multiplying factors for $\mathrm{x}$-axis and $\mathrm{y}$-axis respectively and both are equal to 1 for motion sickness studies; $a_{x, w}(t)$ and $a_{y, w}(t)$ are the frequency-weighted accelerations in the longitudinal and lateral direction. In this work, we adopted the $W_{f}$ by Barnaby and Griffin [36], which is validated and used for weighting lateral acceleration. However, there is no available weighting filter for the longitudinal acceleration or clear guideline could be found. Therefore, in this work, $W_{f}$ for z-direction from ISO 2631-1 [32] is assumed to be the same and apply for longitudinal acceleration. The two weighting curves are shown in Figure 1.

According to the literature [7], a simple linear approximation between $M S D V_{x y}$ and mean passenger illness rating is given as:

$$
I R=K \times M S D V_{x y}
$$

where $I R$ is predicted illness rating and $K$ is an empirically derived constant $(=1 / 50)$ based on data from studies of motion sickness in road [7] and sea [34]. Despite the fact that IR will continue to increase as motion sickness dose value is the integral over time of squared accelerations, this

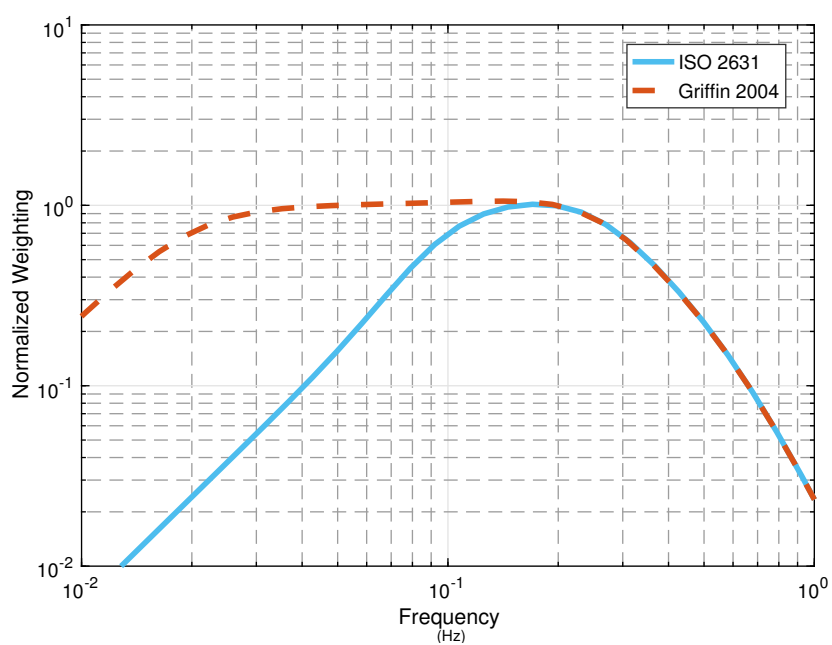

Figure 1: Frequency weighting $W_{f}[32]$, [36].

work is to focus on the minimisation for any given illness rating. The illness rating scale is divided into 4 levels such as 0 indicates feeling fine, 1 indicates slightly unwell, 2 indicates quite ill and 3 indicates absolutely dreadful.

\section{B. Vehicle and Road Tracking Model}

For motion planning, the simplest vehicle model and most commonly used is the point mass model. The vehicle is simplified as a point mass model that can accelerate within bounds. The Equations describing the point mass model are given below (4) :

$$
\ddot{x}=a_{x}, \quad \ddot{y}=a_{y},
$$

where $a_{x}, a_{y}$ are the longitudinal and lateral accelerations. The roads are considered similar to strips and could be described by specifying $x, y$ coordinates of the road centreline and lateral width [37].

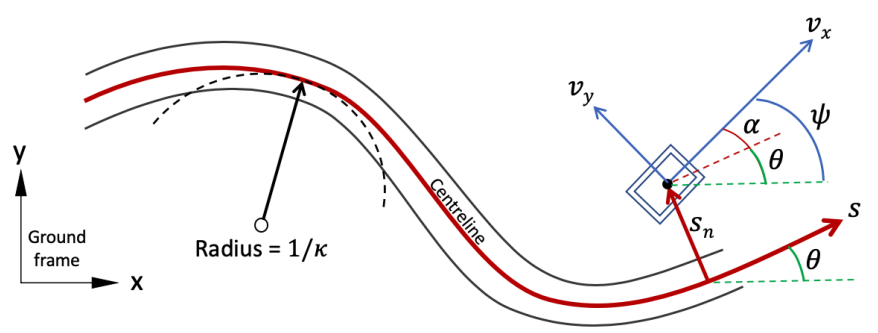

Figure 2: Curvilinear coordinates for road tracking.

The curvilinear coordinates approach have been used in many research works and is the most effective way to describe road centreline using only the line curvature $(\kappa)$ as a function of length $(s),[22]$ as presented in Figure 2. The road heading angle $\theta$ as well as $x, y$ coordinates may be calculated as follows: (5):

$$
\frac{d \theta}{d s}=\kappa(s), \quad \frac{d x}{d s}=\cos \theta, \quad \frac{d y}{d s}=\sin \theta
$$


The main advantage of curvilinear coordinates approach is their usage in tracking the orientation of the vehicle based on forward $\left(v_{x}\right)$ and lateral velocity $\left(v_{y}\right)$ :

$$
\begin{gathered}
\dot{s}=\frac{v_{x} \cos \alpha-v_{y} \sin \alpha}{1-s_{n} \kappa} \\
\dot{s}_{n}=v_{x} \sin \alpha+v_{y} \cos \alpha \\
\dot{\alpha}=\dot{\psi}-\dot{s} \kappa
\end{gathered}
$$

where $\dot{\psi}$ is the yaw rate; $s$ and $s_{n}$ are the longitudinal and lateral position on the road strip; $\alpha$ is the vehicle relative heading to the road. In summary, the vehicle dynamics are describe by means of two control inputs plus eight state variables:

$$
\begin{gathered}
\mathbf{u}=\left[a_{x}, a_{y}\right]^{T} \\
\mathbf{x}=\left[v_{x}, v_{y}, s, s_{n}, \alpha, x, y, \theta\right]^{T}
\end{gathered}
$$

The Equations of motion can be summarised into:

$$
A(\mathbf{x}) \dot{\mathbf{x}}=f[\mathbf{x}(t), \mathbf{u}(t)]
$$

where matrix $A$ is invertible provided that $v_{x}>0$ (i.e., the vehicle never stops), and $s_{n}<1 / \kappa$ (i.e., the vehicle never passes over the local curvature centre of the road)[37].

\section{iII. Optimal Control Problem Formulation}

The problem is to find the appropriate vehicle control inputs, that can drive the vehicle along a predefined path from the initial position $\left(s_{0}\right)$ to the final position $\left(s_{f}\right)$, such that both the motion sickness $(I R)$ and the journey time $(T)$ are minimized. This could be solved as an OCP, by setting specific cost function which represent the objectives and a set of differential equality and algebraic inequality constraints.

\section{A. Problem Formulation}

Following the approach used in many research works in minimum lap time simulation problem [22], it is convenient to change the independent variable from time $(t)$ to distance $(s)$ in the Equations of motion (11). This transformation is based on the following derivation rule:

$$
\dot{x}=\frac{d x}{d t}=\frac{d x}{d s} \frac{d s}{d t}=x^{\prime} \dot{s}=x^{\prime} \gamma
$$

The time domain Equation (11) is then transformed into the distance domain as given in Equation (13):

$$
\gamma A \mathbf{x}^{\prime}=f(\mathbf{x}, \mathbf{u})
$$

The variable change transforms the differential Equation (6) into an algebraic one, that should be eliminated from the Equations of motion in Equation (11). Similarly, the variable $s$ is no longer belong to the state vector. The variable $t$ is re-introduced by writing Equation (6) as (14):

$$
\frac{d t}{d s}=t^{\prime}=\frac{1}{\gamma}=\frac{1-s_{n} \kappa}{v_{x} \cos \alpha-v_{y} \sin \alpha}
$$

Therefore, in $s$ distance domain, the state variables $\mathbf{y}$ and control inputs $\mathbf{u}$ can be summarised as:

$$
\begin{gathered}
\mathbf{y}=\left[v_{x}, v_{y}, s_{n}, \alpha, x, y, \theta, t\right]^{T} \\
\mathbf{u}=\left[a_{x}, a_{y}\right]^{T}
\end{gathered}
$$

The optimal control problem that will be formulated are as follow:

$$
\text { find : } \quad \operatorname{minimize}_{u \in U} \quad J
$$

$$
\begin{array}{r}
\text { subject to : } \quad \gamma A(\mathbf{y}, s) \mathbf{y}^{\prime}=f(\mathbf{y}, \mathbf{u}, s) \\
\phi(\mathbf{y}, \mathbf{u}, s) \leqslant 0 \\
b\left(\mathbf{y}\left(s_{0}\right), \mathbf{y}\left(s_{f}\right)\right)=0
\end{array}
$$

where Equation (17) describes the objective function $J$. Equation (18) describes the Equations of motions and $\gamma>0$, the vehicle never stops, and it cannot revert its direction of travel on the road, $A$ is a non-singular matrix with continuous and piecewise differentiable entries, and it corresponds to the mass matrix of the multi-body model. Equation (19) defines a set of algebraic inequalities $\phi(\mathbf{y}, \mathbf{u}, s)$ involving both the state variables and control inputs. Similarly Equation (20) denotes a set of boundary conditions $b\left(\mathbf{y}\left(s_{0}\right), \mathbf{y}\left(s_{f}\right)\right)$ used to specify the vehicle state at the beginning and at the end of the manoeuvre.

\section{B. Cost Functions}

As mentioned above, our objective is the minimisation of the motion sickness $\left(F_{1}\right)$ but also to obtain solution minimum time problem $\left(F_{2}\right)$.

$$
F_{1}=I R(s) \quad F_{2}=\int_{s_{0}}^{s_{f}} \frac{1}{\dot{s}} d s
$$

To be able to achieve minimum motion sickness without compromising the journey time. The problem is extended and solved as using MOO such that the objective function includes both illness rating and journey time. The Pareto Front approach, where the different targets of the optimization are separated throughout the optimization process and are simultaneously minimized, is utilised in this problem. In this case, the $F_{1}$ and $F_{2}$ in Equation (21) are reformulated into normalized form and given as:

$$
\begin{gathered}
f_{1}=\left(\frac{I R_{\min }}{I R_{\max }-I R_{\min }}\right) F_{1} \\
f_{2}=\left(\frac{T_{\min }}{T_{\max }-T_{\min }}\right) F_{2}
\end{gathered}
$$

where $f_{1}$ and $f_{2}$ are normalised illness rating and journey time. $I R_{\max }$ and $I R_{\min }$ are maximum and minimum illness rating. Similarly, $T_{\max }$ and $T_{\min }$ are the maximum and minimum journey time. Finally, adopting the weighted sum method [38], the contribution of each cost in the combined cost function is controlled through some weighting factors $\left[w_{m}, w_{t}\right]$ as shown below:

$$
J=w_{m} f_{1}+w_{t} f_{2}
$$


with $w_{m} \in[0,1]$ and $w_{t}=1-w_{m}$, where $w_{m}$ and $w_{t}$ are the weighting for the motion sickness weighting and journey time. In theory, $T_{\max }$ would be infinite and $I R_{\min }$ would be (infinitely small) zero. Hence, both $f_{1}$ and $f_{2}$ would always be zero. In this respect, a minimum speed $u_{\text {min }}$ as a boundary is introduced to obtain a baseline value for $T_{\max }$ and $I R_{\min }$ using only motion sickness cost $F_{1}$ in Equation (21) and similarly on the other hand $T_{m i n}$ and $I R_{\max }$ can be obtained based on minimum time problem $F_{2}$ in Equation (21).

\section{Constraints}

In addition, the five inequality constraints in Equations (25, 26, 27, 28 and 29) will be considered, such that the vehicle will be able to accelerate within the bounds Equation (25) set by the friction circle with $a_{\max }$ equal to $1 \mathrm{~g}\left(9.81 \mathrm{~ms}^{2}\right)$, i.e., the maximum absolute acceleration that the vehicle is constrained to reach. The input accelerations are also bounded between minimum acceleration and maximum acceleration given in Equation (26) for longitudinal acceleration and in Equation (27) for lateral acceleration. Also, it will be bounded to never exceed the road borders considering left-width $\left(R_{w}\right)$ and right-width $\left(-R_{w}\right)$ from the centreline of the road Equation (28). Since in a point mass model, the lateral velocity, $v_{y}$ would be zero, hence the only acting velocity is $v_{x}$ is considered as $u$ and it is bounded by a minimum speed $u_{m i n}$ and a maximum speed $u_{\max }$ in Equation(29).

$$
\begin{array}{r}
\sqrt{a_{x}^{2}+a_{y}^{2}} \leqslant a_{\max } \\
a_{x_{\text {min }}} \leqslant a_{x} \leqslant a_{x_{\max }} \\
a_{y_{\min }} \leqslant a_{y} \leqslant a_{y_{\max }} \\
-R_{w} \leqslant s_{n} \leqslant R_{w} \\
u_{\min } \leqslant u \leqslant u_{\max }
\end{array}
$$

Boundary conditions have been added, in order to achieve the best possible optimal solution, with minimum speed is set as $u_{m i n}=5[\mathrm{~m} / \mathrm{s}]$. As far as the road path is concerned, it is generated by a series of curvature $\kappa$ over a distance $s$, to form a path which consists of straight line and curves. From the comparison of different solvers in the literature [39], our optimal control problem has been formulated as described above and solved using GPOPS-II solver [40] with MATLAB suite.

\section{ROAD TRAJECTORY}

In reality, for a specific starting point there would be a number of routes the vehicle could travel to arrive at the final destination. The motion sickness and journey time would also depend on the route taken such as in some cases a short route with many turns might save journey time but would result in higher motion sickness incidence. Similarly, longer route would take longer to arrive with less sickness. The study [7] also found that motion sickness incidence is greater in countryside road due to higher magnitudes of lateral vehicle motion compared to motorway where

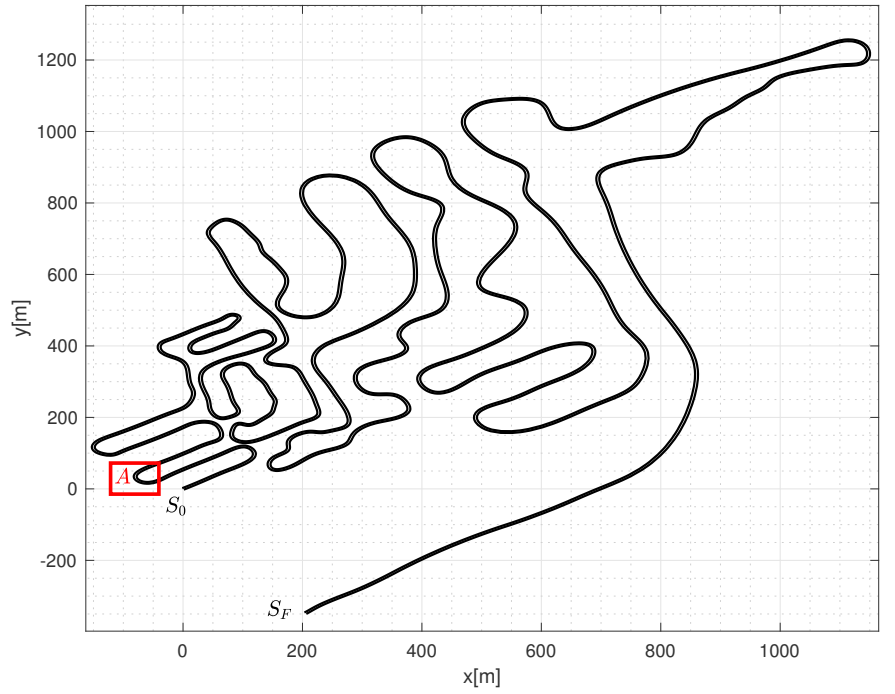

Figure 3: Road path containing different road characteristic where $S_{0}$ is the starting point and $S_{f}$ is the finishing point. The red box A will be used to illustrate the trajectory for differences road width study in section $\mathrm{V}-\mathrm{C}$.

duration of travel is lower. This paper aims to capture different road characteristic that are included in urban, motorway and countryside road (i.e., a straight road, and turns with high and low curvature). In this respect, a road path, which includes the combination of such characteristics is shown in Figure 3. The road is $12.2 \mathrm{~km}$ long and it is selected for its duration to be such to extract concrete conclusions for the motion sickness. In addition, the road is assumed to be one way lane with centreline as well as left and right border in which vehicle is able to manoeuvre within the road bound. Also, the road is flat with smooth surface as no suspension dynamics is considered. Finally, no obstacle or disturbance along the road journey as this paper presents an offline optimization solutions.

\section{Results}

The application of motion planning using optimal control is utilized to mitigate motion sickness in autonomous vehicles. More specifically, for a predefined road path from a specific starting point to a final one, the optimum trajectory is sought within specific and given boundary and constraints in order to minimize the motion sickness without compromising the journey time. The trade-off between illness rating and journey time has been extensively studied (24) through a set of weighting factors $\left[w_{m}, w_{t}\right]$. In this respect, the weighting factor for the motion sickness term $w_{m}$ is varied from 0 to 1 with increment of 0.05 . In addition, three case studies are then carried out to investigate the effect of driving styles, maximum driving speed, and road width on the trade-off between illness rating and journey time. More specifically 
1) For driving style, combinations of weighting factors are selected to simulated different driving styles; ranging from sport, natural, comfort and antinausea.

2) For vehicle speed, the maximum allowed speed that the vehicle can drive is varying and its effect on motion sickness is investigated.

3) For road width limit, fixed path and free path scenarios are compared. For fixed path, where the road doesn't allow any lateral manoeuvrability to the vehicle by setting the road width at zero (i.e., the road boundary of left border and right border measured from the centreline $R_{w}=0$. On the other hand, in the free path cases, the road width is increased as $R_{w}>0$.

The solutions for all the case studies are illustrated and discussed in detail together with figures and tables in section V-A, V-B and V-C. In Figure 4, the cumulative illness rating curve over the journey time for each set of weighting factor are plotted together forming a Pareto front. From the same Pareto front, the driving style analysis is performed with selected cases $\left[w_{1}, w_{2}\right.$, $\left.w_{3}, w_{4}\right]$. In addition, the frequency analysis is carried out using Matlab command: "fft" to investigate their frequency weighted acceleration power spectral densities given in Figure 5. The optimal motion profile for these driving styles are given in Figure 6. Similarly for vehicle driving speed $\left[u_{1}, u_{2}, u_{3}, u_{4}\right]$ and road width $\left[R_{w 1}, R_{w 2}\right.$, $R_{w 3}$ ] studies, Pareto fronts obtained for these conditions are given in Figure 7 and 9 respectively, the frequency analysis in Figure 8 and 11. In addition, the optimum trajectories of the road width cases are shown in Figure 10. The illness rating and respective journey time for each study are tabulated in Table I, II and III.

\section{A. Driving styles}

In this part of the study, the condition for speed limit is set at $u_{\max }=30[\mathrm{~m} / \mathrm{s}]$ with road width limit of $R_{w}=1.5 \mathrm{~m}$. The cumulative illness rating over the journey time for the set of weighting factors is shown in Figure 4. The illness rating curve decreases when there is an increase in weighting $w_{m}$ as the objective function would also prioritize illness rating. However, at the same time it results in longer journey time. When plotting all the cumulative illness rating curves together, a complete Pareto front is obtained in Figure 4. This front presents the correlation between the minimum motion sickness with the duration of travel. It is obvious that shortest journey time would results in higher motion sickness. Therefore, the compromise between the two objectives should identified based on the preference of the user.

In this respect, the optimal solutions from four combination of weighting factors are selected $\left[w_{1}, w_{2}, w_{3}, w_{4}\right]$ and

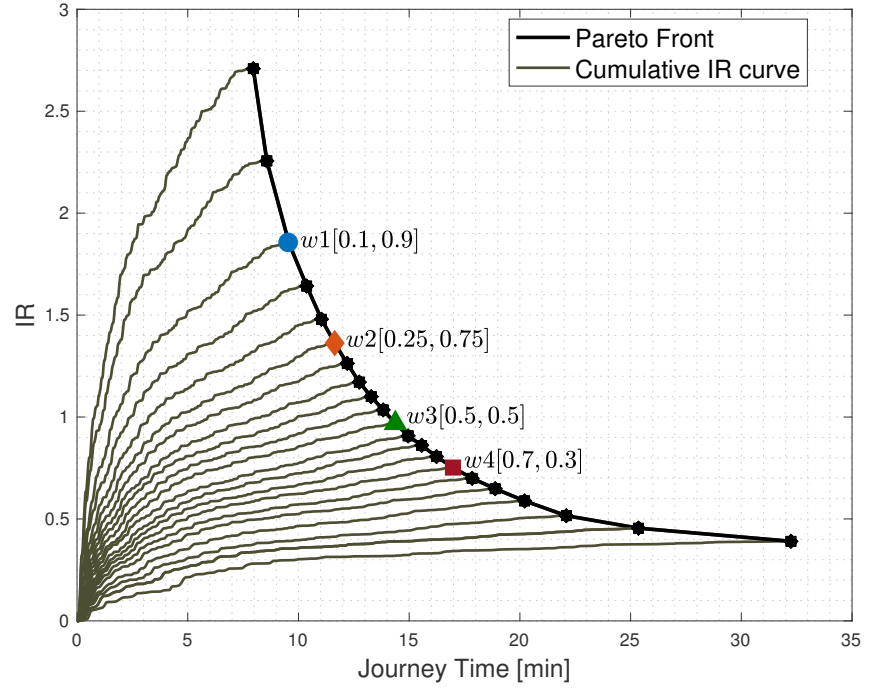

Figure 4: Cumulative illness rating curve for each weighting pair forming a Pareto front and showing selected weighting pair for four driving styles.

tabulated in Table I. In the near future, the automated vehicle will be able to allow the passenger to select a particular driving style based on their preference and taking into consideration the arrival time and the induced motion sickness levels. Thus, the weighting pairs from the optimal Pareto front could be used to represent the different automated driving styles of the same vehicle such that $w_{1}$ can be used to represent the Style1, $w_{2}$ for Style2, $w_{3}$ for Style3, and $w_{4}$ for Style4. From Table I, it is clear that when $w_{m} \gg w_{t}$, the objective would favour the need to reduce motion sickness and compromise the journey time which is Style4. On the other hand for $w_{m} \ll w_{t}$, journey time is favoured and in this case Style 1 as shown in Figure 4. In this way the optimal solution is found by varying the weighting factors in order to achieve minimum motion sickness without comprising journey time.

Table I: Comparison of IR and Journey time between four driving styles.

\begin{tabular}{|c|c|c|c|}
\hline & {$\left[w_{m}, w_{t}\right]$} & $I R$ & Time $[$ min $]$ \\
\hline Style 1 & {$[0.1,0.9]$} & 1.85 & 9.6 \\
\hline Style 2 & {$[0.25,0.75]$} & 1.36 & 11.6 \\
\hline Style3 & {$[0.5,0.5]$} & 0.97 & 14.4 \\
\hline Style4 & {$[0.7,0.3]$} & 0.75 & 17.0 \\
\hline
\end{tabular}

Additionally, frequency analysis is carried out on the resulting accelerations for each driving mode by investigating their power spectral densities shown in Figure 5. The trend of the acceleration power spectra is similar for all the driving style while the acceleration spectral in $\mathrm{x}$ and y-axis are in the range below $0.5 \mathrm{~Hz}$. Both spectra have a peak at around $0.1-0.18 \mathrm{~Hz}$. According to Figure 5 , lateral acceleration appear to be dominating with higher r.m.s values compared to the longitudinal acceleration due to longer winding road sections. The peak in sport driving 
is gradually suppressed when switching into other styles especially in driving Style4, where the peak is minimum. This is because in driving Style1, the acceleration and braking driven by AV would be harsher than any other driving style, especially when initiating the turn, which results in higher lateral acceleration. Similarly in the case of driving Style $3, \mathrm{AV}$ drives in a gentle manner considering passenger comfort and avoid motion sickness, but at the same time maintaining the journey time to minimum.

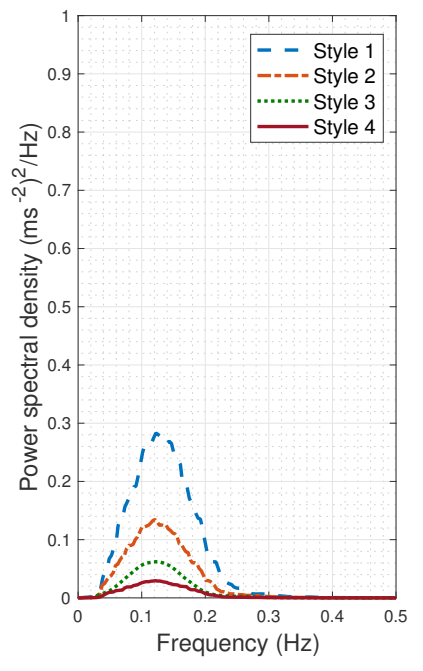

(a) $\mathrm{x}$-axis

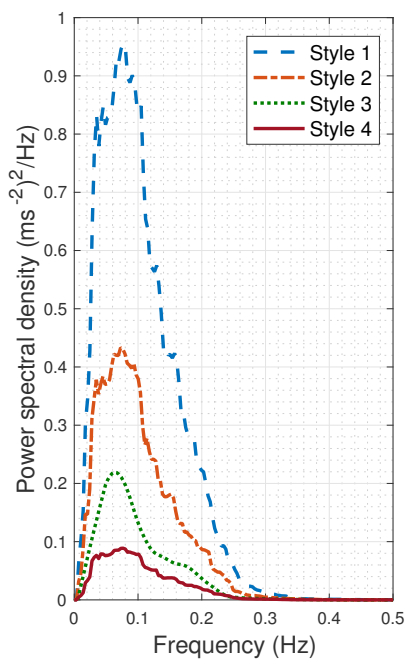

(b) y-axis
Figure 5: Frequency weighted acceleration power spectral densities in the longitudinal and the lateral axis for four driving styles.

In addition the optimum motion profiles, given in Figure 6 also reflect the behaviour of each driving style with highest driving speed in driving Style1 compared to the other styles The velocity profile in Style 3 is in a smoother transition throughout the journey and prevents vehicle moving too fast or too slow. The velocity profile for all driving style shows similar forms by driving faster at straight road and slow down before initiating the turn and speed up after leaving the turn.

\section{B. Vehicle speed}

In section $\mathrm{V}$-A, it is understood that different driving styles would result in different velocity profiles. However, this case study aims to investigate the influence of the vehicle speed on motion sickness which is based on the maximum allowed speed that the vehicle can drive. So that the vehicle is restricted by $u_{\max }$. In this respect, the maximum speed is varied and set as $u_{\max }=\left[u_{1}, u_{2}\right.$, $\left.u_{3}, u_{4}\right] \mathrm{m} / \mathrm{s}$ on the same road width at $R_{w}=1.5 \mathrm{~m}$. So, through the variation of $u_{\max }$, the study will focus on motion sickness based on different vehicle speeds.

In this respect, we assigned $u_{1}=20 \mathrm{~m} / \mathrm{s}$ as $V_{\max _{1}}, u_{2}=$ $25 \mathrm{~m} / \mathrm{s}$ as $V_{\text {max }_{2}}, u_{3}=30 \mathrm{~m} / \mathrm{s}$ as $V_{\max _{3}}$ and $u_{4}=40 \mathrm{~m} / \mathrm{s}$ as

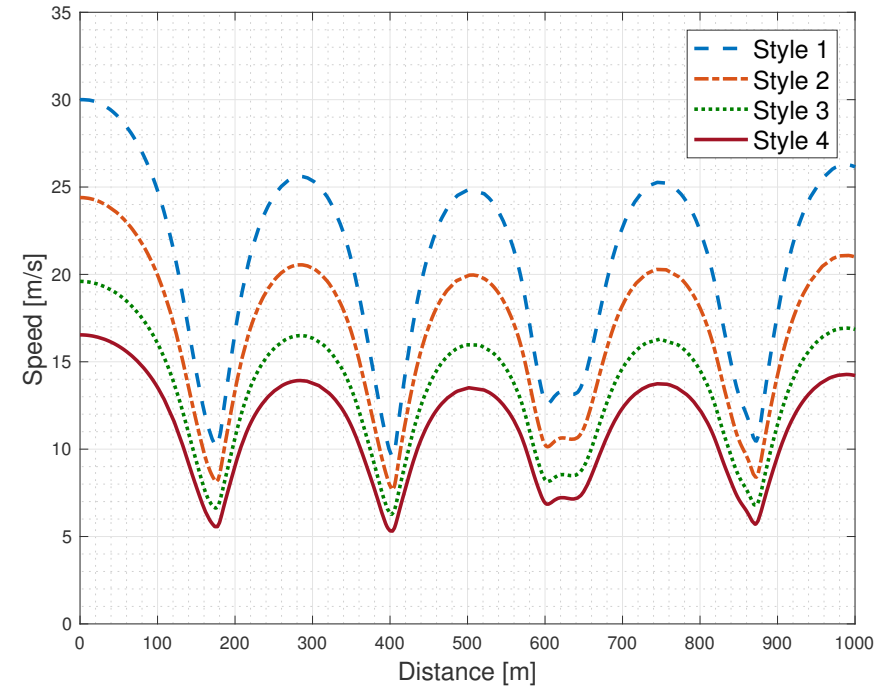

Figure 6: The optimum velocity profile along the road journey for four driving styles.

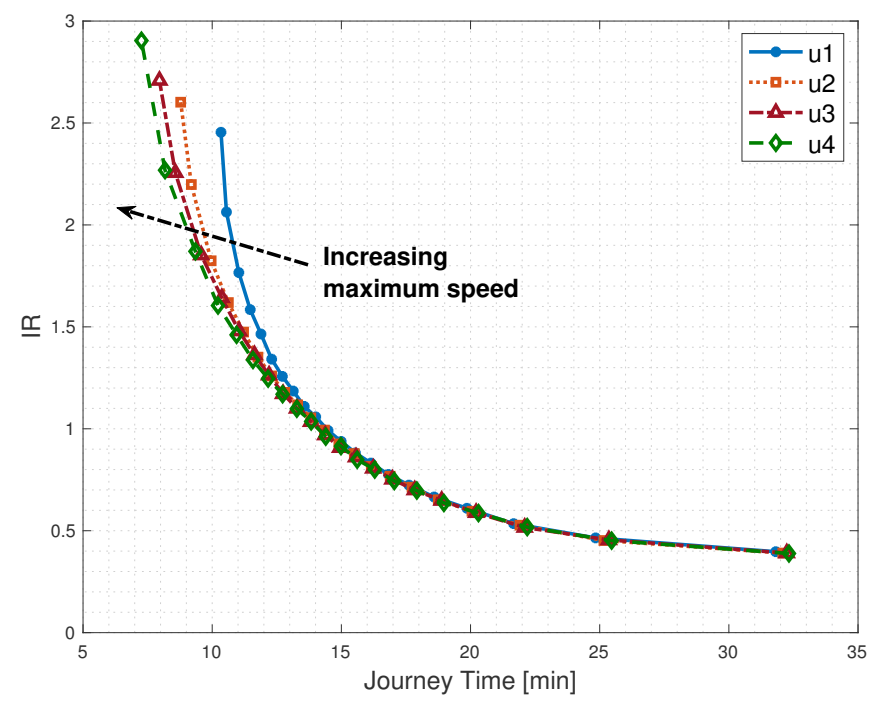

Figure 7: Pareto fronts for four driving speed showing large variation when $w_{m} \ll w_{t}$.

$V_{\max _{4}}$. Similarly based on Equation (24), a Pareto front is achieved through a set of weighting factors $\left[w_{m}, w_{t}\right]$ for each vehicle. The Pareto fronts for all four $V_{\max }$ are presented in Figure 7 containing the relationship between illness rating and journey time based on all the weighting factors. For $w_{m} \ll w_{t}$, there is a large variation between the fronts due to the fact the vehicle is able to reach higher driving speed. On the other hand, the variation becomes smaller and all the fronts converge to the same curve, when $w_{m} \gg w_{t}$ as the vehicle is now driving as slow as possible to favour motion sickness in the cost function.

In order to investigate in depth, the driving Style 1 is selected to be compared for all $V_{\max }$. The respective illness rating and journey are given in Table II. It can be 
seen that $V_{\max _{1}}$ with the lowest vehicle speed results in longest journey time compared to other three vehicles. On the other hand $V_{\max _{4}}$ which can exert highest vehicle speed leads to fastest journey time. Although, there is no significant differences in term of sickness, the journey time could be save up to one and a half minute by adjusting the vehicle speed.

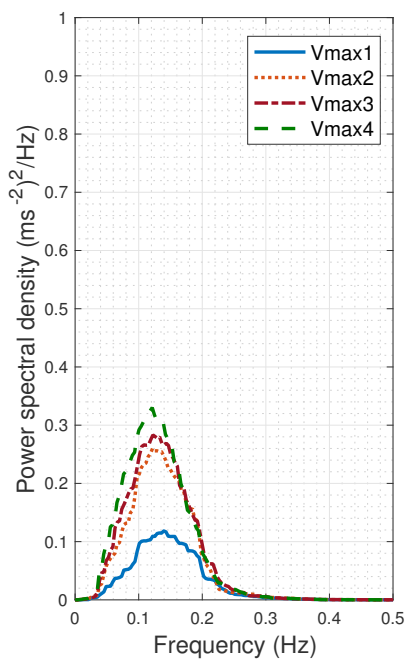

(a) $\mathrm{x}$-axis

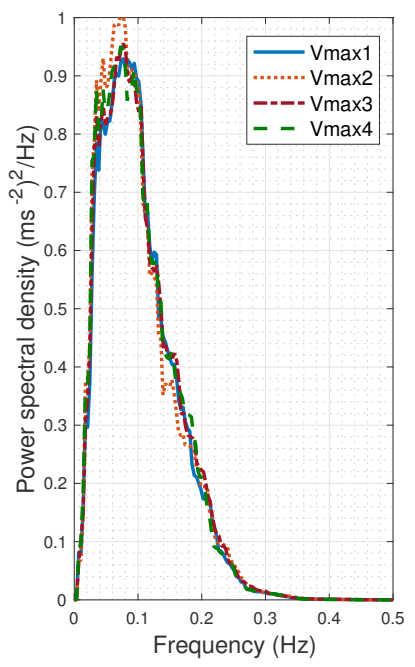

(b) y-axis
Figure 8: Frequency weighted acceleration power spectral densities in the longitudinal and the lateral axis for four $V_{\max }$ in driving Style1.

Table II: Comparison IR and Journey time between four $V_{\max }$ in driving Style1.

\begin{tabular}{|c|c|c|}
\hline & $I R$ & Time $[$ min $]$ \\
\hline$V_{\max _{1}}$ & 1.77 & 11 \\
\hline$V_{\max _{2}}$ & 1.824 & 10 \\
\hline$V_{\max _{3}}$ & 1.85 & 9.6 \\
\hline$V_{\max _{4}}$ & 1.87 & 9.4 \\
\hline
\end{tabular}

Similarly, by looking at the power spectral densities for both acceleration shown in Figure 8, the variation in lowfrequency motion is mainly in fore-and-aft acceleration due to the fact that the differences in accelerating and braking performance affected by the vehicle speed capabilities. The peak in $V_{\text {max }_{3}}$ and $V_{\text {max }_{4}}$ is higher in x-axis, but slightly lower in $y$-axis compared to the rest. This reflects the nature of the road, as at straight road section, the vehicle with $V_{\max _{3}}$ and $V_{\max _{4}}$ are able to drive at higher speeds to compensate the journey time. Interestingly, it could be assumed that each $V_{\max }$ represent a vehicle from the perspective of the maximum speed capabilities. Since from the literatures [7] and [8], other vehicle dynamics such as chassis, tire or suspension dynamics are known to have less or no significant influence on motion sickness.

\section{Road width}

In [27] and [29], their road width study shown that the vehicle could utilize the available road width to achieve lower sickness while maintaining shorter journey time compared to travelling on a fixed road. However, the effect of different road characteristic on a longer road were not considered. Therefore, the aim of this section is to investigate whether the vehicle could take the advantage of road width when the road is extended to include different road characteristics as well as longer route journey. In this part of the study, the condition for speed limit is set at $u_{\max }=30[\mathrm{~m} / \mathrm{s}]$, but the road width limit of $R_{w}$ is set to vary. Thus, for the same vehicle driving speed, a fixed road path $\left(R_{w}=0\right)$ and free road paths where $\left(R_{w}>0\right)$ are selected for the analysis such that $R_{w 1}=0 \mathrm{~m}, R_{w 2}=1.5 \mathrm{~m}$ and $R_{w 3}=2.5 \mathrm{~m}$.

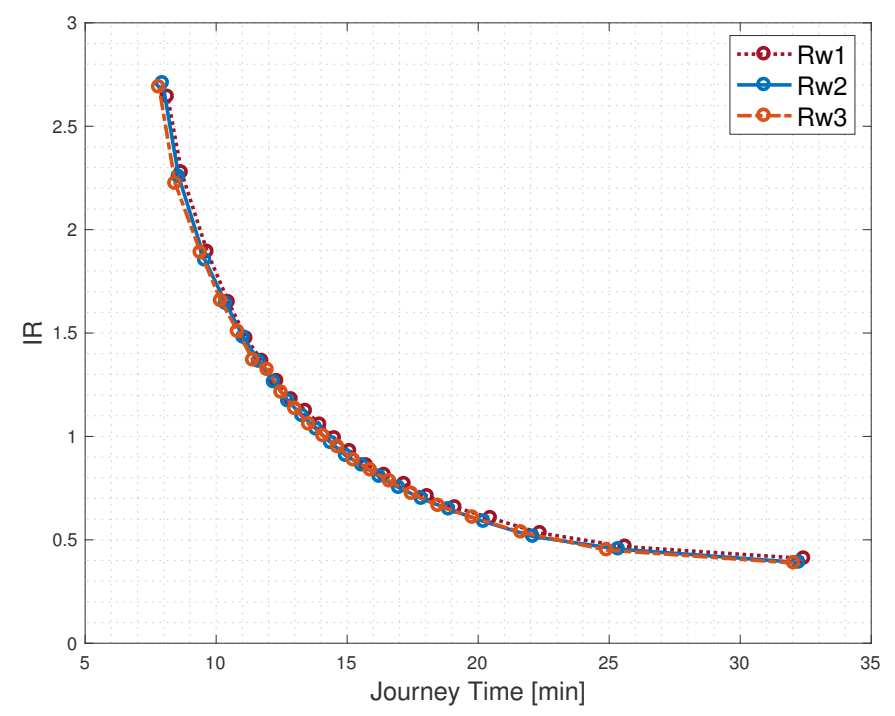

Figure 9: Pareto fronts for three road width cases with same speed limit $u_{3}$.

Table III: Comparison IR and Journey time between three road width cases using driving Style3.

\begin{tabular}{|c|c|c|}
\hline & IR & Time $[$ min $]$ \\
\hline$r_{1}$ & 1.37 & 11.8 \\
\hline$r_{2}$ & 1.37 & 11.6 \\
\hline$r_{3}$ & 1.36 & 11.4 \\
\hline
\end{tabular}

Using the same approach as in sections $\mathrm{V}-\mathrm{A}$ and $\mathrm{V}-\mathrm{B}$, the Pareto front from each road path is shown in Figure 9. The variation between the fronts in road width study is significantly small. Nevertheless, it can be seen that the front shift slightly downward when the road width is increased to allow lateral manoeuvrability however it is notably at $w_{m} \ll w_{t}$ region. This is due to the fact that when the road is longer, the advantage of road width become less since any loss in journey time would average out when travelling on different road characteristics when 
$w_{m} \gg w_{t}$.

To put this into a perspective, the driving Style3 (i.e., $\left.w_{3}\right)$ is selected to be compared for all the road scenarios. This is presented as $r_{1}, r_{2}$ and $r_{3}$ accordingly. The improvement of the result significantly small and echoed in the low-free frequency spectra shown in Figure 11. A section of the road shown in Figure 10 illustrated that for fixed scenario, the vehicle travels on the centreline, whereas for free path scenarios, especially in $r_{3}$, the vehicle is able to utilise the road width for a smaller turning radius. However, in this case study, the road width contribution to sickness and journey time is negligible when travelling on a long road journey.

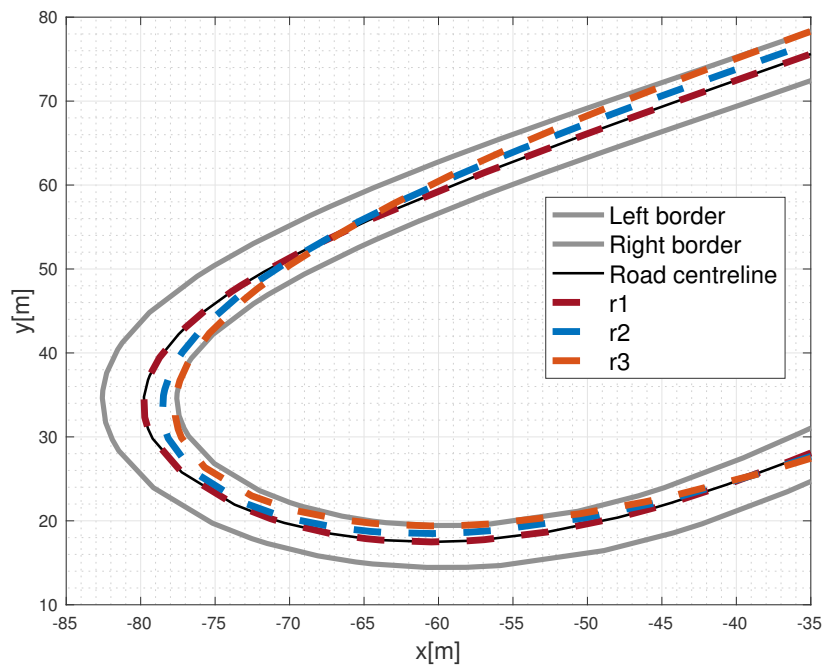

Figure 10: The optimum road trajectory in road segment (A) for three road cases.

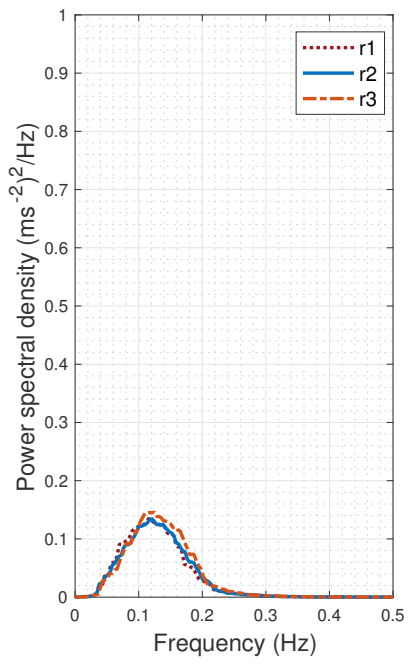

(a) $\mathrm{x}$-axis

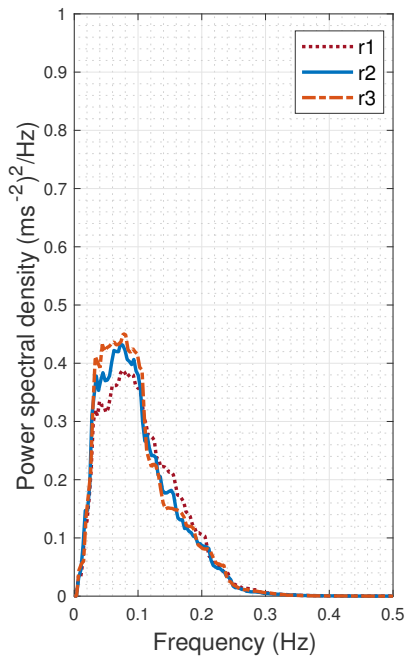

(b) y-axis
Figure 11: Frequency weighted acceleration power spectral densities in the longitudinal and the lateral axis for road width study using driving Style 3 .

\section{Discussion}

The trade-off between motion sickness and journey time is investigated using multi-objective optimization in the OCP problem to compute best comprise solutions. This is done through the application of weighted sum method by varying the weighting to each objective included in the cost function. The resulting Pareto fronts in all case studies are similar, and no convex solution can be found. This indicates the efficiency of the weighing sum method applied in this work. The Pareto front in each case study suggests that the trade-off between the two objectives is reachable among the best comprise solutions within the optimal front. In addition, the solution obtained from the weighting pairs in the optimal Pareto front have been used to exam the effect of the driving style. In the driving styles case study, it can be seen that driving style is regulated by the weighting pair. As expected, the driving style which aims for lower journey time, results in higher intensity to the optimal motion profiles causing more sickness. On the other hand, the optimal motion profiles have lower intensity when the weighting favours motion sickness in the cost function.

From all the case studies, Table I and Figure 5 provide a clear evidence that driving style is the primary factor influencing the low frequency longitudinal and lateral motion in road vehicles. This is consistent with the findings in in the literature [8] as driving style from the driver dictates the motion profiles for the whole journey. In the second case study, the effect of different vehicle speed capability is investigated by varying the maximum speed. According to Table II, the journey time can be decreased up to 90 seconds for a relatively similar illness rating between the four vehicle maximum speed. However, the effectiveness is significant only when the weighting for journey time is higher in the optimization. The effect of the vehicle speed capability reduces when the weighting for motion sickness is higher. Finally, in the last case study, the influence of the road width becomes negligible when travelling on longer journey. As far as the spectra are concerned, in all the case studies they show similarities between the longitudinal and lateral accelerations. However, the spectra for lateral direction is higher than the longitudinal direction due to the fact that the road path used in this work consists of many winding road sections.

\section{FUTURE WORK}

In addition to the important outcomes in this paper, many questions still remain to be addressed as there are several modelling assumptions and simplification, without considering any disturbance from external agents. Thus, the optimal trajectory returned by the motion planner from open-loop references is unlikely to be exactly reproduce by real-world vehicles. Therefore, a robust openloop trajectory is desired in order to enhance the correlation between numerical-control and real-world driving. 
The robustness of such trajectory could be achieved by considering a selection of inequality constraints involved in the context of motion sickness. This would allow the margin of error when uncertainties or disturbances were to appear. This can be done by incorporating higherfidelity vehicle and motion sickness models in the OCP problem and including additional constraints for obstacles avoidance; traffic light; lane-changing; stop-and-go; and overtaking scenarios. In this way, their effect on motion planning for motion sickness minimization could be studied further. Additionally, It is also crucial to investigate whether the low-level controllers would be able to track the reference optimal trajectory. Finally, the experimental work is required to carry out in order to investigate the validity of the optimal trajectory generated by the numerical approach.

\section{Conclusions}

The contribution of this paper is the fact that motion sickness can be minimized through the application of motion planning through optimal control method. The MOO method is used in the OCP to obtain various alternatives that compromise optimally motion sickness and journey time. The Pareto front representing the correlation between the two components is obtained and this front also allows user to select their preference driving style. From the three case studies, driving styles have a bigger impact on reducing motion sickness and journey time rather than vehicle speed and the road width. There is a significant impact in vehicle speed when $w_{m} \ll w_{t}$, such that all the vehicles result in similar sickness level, but journey time is shortened for vehicle with higher maximum speed capabilities. However, the effect of road width is negligible when travelling on longer road for the reduction of motion sickness and journey time. This finding is crucial considering the need for automated vehicles to drive on a fixed road path in respect to road safety and also to allow the employment of connected and automated vehicles in the future.

\section{ACKNOWLEDGMENT}

The research is partially funded by Innovate UK through the AIDCAV project and fully funded by the EPSRC under Grant EP/N509450/1.

\section{REFERENCES}

[1] Litman, T., "Autonomous vehicle implementation predictions: Implications for transport planning ". 2020.

[2] Sivak, Michael, and Brandon Schoettle. "Motion sickness in selfdriving vehicles." 2015.

[3] Wada, Takahiro. "Motion Sickness in Automated Vehicles." Advanced Vehicle Control: Proceedings of the 13th International Symposium on Advanced Vehicle Control (AVEC'16), September 13-16, 2016, Munich, Germany. CRC Press, 2016.

[4] Diels, C. and Bos, J.E., 2015. "Design guidelines to minimise self-driving carsickness". In Automated Vehicles Symposium.

[5] Griffin, Michael J. "Handbook of human vibration". Academic press, 2012.

[6] Cheung, B., and Nakashima, A. "A review on the effects of frequency of oscillation on motion sickness". No. DRDC-TR-2006229. Defence research and development Toronto (Canada),2006.
[7] Turner, Mark, and Michael J. Griffin. "Motion sickness in public road transport: the effect of driver, route and vehicle."Ergonomics 42.12 (1999): 1646-1664.

[8] Griffin, M.J. and Newman, M.M., "An experimental study of low-frequency motion in cars". Proceedings of the Institution of Mechanical Engineers, Part D: Journal of Automobile Engineering, 2004. 218(11), pp.1231-1238.

[9] Elbanhawi, Mohamed, Milan Simic, and Reza Jazar. "In the passenger seat: investigating ride comfort measures in autonomous cars." IEEE Intelligent Transportation Systems Magazine 7.3 (2015): 4-17.

[10] Diels, Cyriel, and Jelte E. Bos. "Self-driving carsickness." Applied ergonomics 53 (2016): 374-382.

[11] Diels, Cyriel, et al. "Motion sickness in automated vehicles: the elephant in the room. Road Vehicle Automation 3. Springer International Publishing, 2016. 121-129.

[12] Papaioannou G, Voutsinas A, Koulocheris D. "Optimal design of passenger vehicle seat with the use of negative stiffness elements." Proceedings of the Institution of Mechanical Engineers, Part D: Journal of automobile engineering. 2020 Feb;234(23):610-29.

[13] Salter S, Diels C, Herriotts P, Kanarachos S, Thake D. "Motion sickness in automated vehicles with forward and rearward facing seating orientations." Applied ergonomics. 2019 Jul 1;78:54-61.

[14] Wada, Takahiro, Satoru Fujisawa, and Shunichi Doi. "Analysis of driver's head tilt using a mathematical model of motion sickness."International Journal of Industrial Ergonomics (2016).

[15] Salter S, Thake D, Kanarachos S, Diels C. "Motion Sickness Prediction Device for Automated Vehicles. "International Journal of Mechanical and Production Engineering. 2019 May $21 ; 7(2): 68-74$

[16] Wu, Z., Liu, Y. and Pan, G., "A smart car control model for brake comfort based on car following". IEEE transactions on intelligent transportation systems, 2009. 10(1), pp.42-46.

[17] Papaioannou, G. and Koulocheris, D. , "An approach for minimizing the number of objective functions in the optimization of vehicle suspension systems". Journal of Sound and Vibration, 2018. 435, pp.149-169.

[18] Hiraoka T, Kunimatsu T, Nishihara O, Kumamoto H. Modeling of driver following behavior based on minimum-jerk theory. InProc. 12th World Congress ITS 2005 Nov.

[19] Bast, H., Delling, D., Goldberg, A., Müller-Hannemann, M., Pajor, T., Sanders, P., Wagner, D. and Werneck, R.F. "Route planning in transportation networks". In Algorithm engineering (pp. 19-80). 2016. Springer, Cham.

[20] Brechtel S., Gindele T., and Dillmann R., "Probabilistic MDP behavior planning for cars," in 14th International Conference on Intelligent Transportation Systems, pp. 1537?1542, IEEE, 2011.

[21] Paden B, Čáp M, Yong SZ, Yershov D, Frazzoli E. " A survey of motion planning and control techniques for self-driving urban vehicles. "IEEE Transactions on intelligent vehicles. 2016 Jun $13 ; 1(1): 33-55$.

[22] Smith EN, Velenis E, Tavernini D, Cao D. "Effect of handling characteristics on minimum time cornering with torque vectoring." Vehicle system dynamics. 2018 Feb 1;56(2):221-48.

[23] R. S. Sharp \& Huei Peng (2011) "I Vehicle dynamics applications of optimal control theory," Vehicle System Dynamics, 49:7, 1073-1111, DOI: 10.1080/00423114.2011.586707

[24] Hult R, Tabar RS. "Path planning for highly automated vehicles" . Mathesis, Gothenburg. 2013.

[25] Förstberg J. "Ride comfort and motion sickness in tilting trains", Doctoral dissertation, KTH Royal Institute of Technology, 2000.

[26] H. Dong, L. Zhang, Y. Chen, et al. "Multi-objective train trajectory design based on dynamic programming." Proceedings of the Chinese Control Conference. Nanjing: IEEE, 2014.

[27] Htike Z, Papaioannou G, Velenis E, Longo S. "Motion Planning of Self-driving Vehicles for Motion Sickness Minimisation." In2020 European Control Conference (ECC) 2020 May 12 (pp. 1719-1724). IEEE.

[28] Htike Z, Papaioannou G, Siampis E, Velenis E, Longo S. "Motion Sickness Minimisation in Autonomous Vehicles Using Optimal Control." In International Conference on Robotics in Alpe-Adria Danube Region 2020 Jun 19 (pp. 275-282). Springer, Cham.

[29] Htike, Z., Papaioannou, G., Siampis, E., Velenis, E. and Longo, S., "Minimisation of Motion Sickness in Autonomous Vehicles." 
In 2020 IEEE Intelligent Vehicles Symposium (IV) (pp. 11351140). IEEE.

[30] Becerra, I., Suomalainen, M., Lozano, E., Mimnaugh, K.J., Murrieta-Cid, R. and LaValle, S.M.,. 'Human perceptionoptimized planning for comfortable vr-based telepresence." IEEE Robotics and Automation Letters, 5(4), pp.6489-6496.

[31] British Standards Institution 1987, "British standard guide to measurement and evaluation of human exposure to whole-body mechanical vibration and repeated shock," British Standards Institution, London.

[32] International Organization for Standardization, 1997. "Mechanical vibration and shock-Evaluation of human exposure to wholebody vibration-Part 1: General requirements." The Organization.

[33] Bles, W., Bos, J.E., De Graaf, B., Groen, E. and Wertheim, A.H. "Motion sickness: only one provocative conflict?". Brain research bulletin, 1998, 47(5), pp.481-487.

[34] Lawther, A. and Griffin, M.J., 1986. "The motion of a ship at sea and the consequent motion sickness amongst passengers." Ergonomics, 29(4), pp.535-552.

[35] ISO 8041, " Human response to vibration - Measuring instrumentation". 2005

[36] Donohew, Barnaby E., and Michael J. Griffin."Motion sickness: effect of the frequency of lateral oscillation." Aviation, Space, and Environmental Medicine 75.8 (2004): 649-656.

[37] Lot R, Biral F. "A curvilinear abscissa approach for the lap time optimization of racing vehicles." IFAC Proceedings Volumes. 2014 Jan 1;47(3):7559-65.

[38] Marler, R.T. and Arora, J.S., 2010. " The weighted sum method for multi-objective optimization: new insights.Structural and multidisciplinary optimization, 41(6), pp.853-862.

[39] Dal Bianco, N., Bertolazzi, E., Biral, F. and Massaro, M.. “ Comparison of direct and indirect methods for minimum lap time optimal control problems". Vehicle System Dynamics, 2019. 57(5), pp.665-696.

[40] Patterson MA, Rao AV. " GPOPS-II: A MATLAB software for solving multiple-phase optimal control problems using hpadaptive Gaussian quadrature collocation methods and sparse nonlinear programming. "ACM Transactions on Mathematical Software (TOMS). 2014 Oct 27;41(1):1.

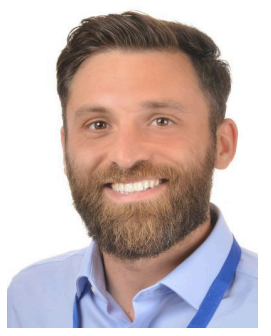

Stefano Longo (Senior Member, IEEE) received the M.Sc. degree in control systems from the University of Sheffield in 2007 and the Ph.D. degree in networked control systems from the University of Bristol in 2011. In November 2010, he was appointed to the position of Research Associate at Imperial College London. He is currently a Senior Lecturer (an Assistant Professor) in vehicle control with Cranfield University since 2012. He is the Course Director for the M.Sc. in automotive mechatronics and advanced motorsport mechatronics, a Chartered Engineer, an Associate Editor of Mechatronics Journal (Elsevier), an Elected Executive Member of IET Control and Automation Network and IET Automotive and Road Transport Systems Network, and a member of the IFAC Technical Committee on Mechatronic Systems and Automotive Control. In the last few years he has authored two books and over 70 research articles in peer-refereed journals and international conferences. His work and his research interests gravitate around the problem of implementing advanced control algorithms in hardware, where the controller design and the hardware implementation are not seen as two separate, decoupled problems, but as a whole. His Ph.D. thesis was awarded the Institution of Engineering and Technology (IET) Control and Automation Prize for significant achievements in the area of control engineering.

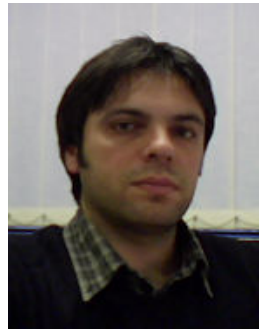

Efstathios Velenis received the Ph.D. degree from the School of Aerospace Engineering, Georgia Institute of Technology (Georgia Tech), Atlanta, GA, USA, in 2006. He is currently a Senior Lecturer with the Advanced Vehicle Engineering Centre, Cranfield University, Cranfield, U.K. He obtained research funding from EPSRC, Innovate U.K., and the European Commission. His current research interests include vehicle dynamics and control, optimal control for active chassis systems, traction, braking, and handling control for electric/hybrid vehicles, lap-time optimization, and tire modeling. Dr. Velenis was a recipient of the Luther Long Award for best Ph.D. dissertation in the area of engineering mechanics from Georgia Tech.

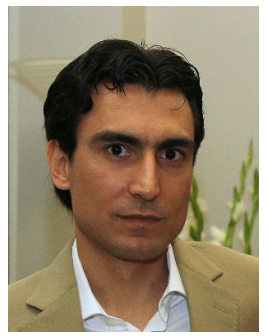

Efstathios Siampis Dr Efstathios Siampis joined the AVEC of $\mathrm{CU}$ as a Lecturer in December 2019. He received his PhD degree from AVEC in 2017 for his work on predictive torque-vectoring control for EVs, which resulted in several journal and conference papers. Following his PhD, he joined Delta Motorsport as a Vehicle Dynamics and Control Engineer and worked on several research (APC Hipercar in collaboration with Ariel Motor Co., AIDCAV) and commercial projects. He is currently a Co-Investigator of the "AIDCAV" project, supervising a team of 3 post-doctoral researchers and $2 \mathrm{PhD}$ students. His research interests include vehicle dynamics modelling, simulation, optimal control, estimation and embedded systems..

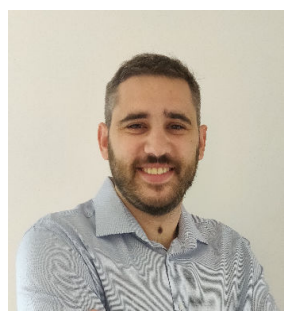

Georgios Papaioannou Georgios Papaioannou is a Postdoctoral Researcher at KTH Royal Institute of Technology in Sweden, while this work was conducted as a Research Fellow at Cranfield University. He received his $\mathrm{PhD}$ from School of Mechanical Engineering at National Technical University of Athens (NTUA), Greece in 2019. His PhD work focused on the optimization of vehicle and seat suspension systems, prompting novel suspension systems and optimization methods, and received the second Jacob Giourounlian award for its potential industrial impact from NTUA. His research interests include automated and human driven vehicles, vehicle dynamics, human factors, motion comfort, suspension design, seat design, tire modelling, optimization, control and motion planning.

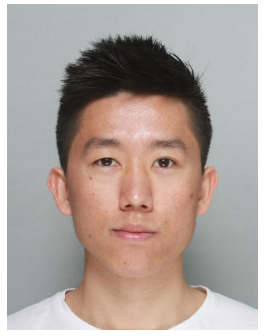

Zaw Htike is a $\mathrm{PhD}$ student at Cranfield University. He received his MSc degree in Aerospace Dynamics from Cranfield University. He did his undergraduate degree at the University of Bristol in Aerospace Engineering. He was a member of KTH Formula student, working in the Aerodynamics Design and Manufacturing team during an Erasmus year at KTH Royal Institute of Technology. His research interests focus on the application of optimal control for minimising motion sickness 
$2021-12-28$

Fundamentals of motion planning for mitigating motion sickness in automated vehicles

Htike, Zaw

IEEE

Htike Z, Papaioannou G, Siampis E,et al., (2022) Fundamentals of motion planning for

mitigating motion sickness in automated vehicles. IEEE Transactions on Vehicular Technology,

Volume 71, Number 3, March 2022, pp. 2375-2384

https://doi.org/10.1109/TVT.2021.3138722

Downloaded from Cranfield Library Services E-Repository 